\title{
Hierachical Porous Carbon Structures from Cellulose Acetate Fibers
}

\begin{abstract}
S. Polarz, ${ }^{*, t, \ddagger}$ B. Smarsly, ${ }^{\dagger, \S}$ and J . H. Schattka ${ }^{\dagger}$
Lash Miller Chemical Laboratories, University of Toronto, 80 St. George Street, Toronto, Ontario M5S 3H6, Canada, Max-Planck Institute of Colloids and I nterfaces, Research Campus Golm, 14424 Potsdam, Germany, and Advanced Material Laboratory, University of New Mexico, 1001 University Boulevard SE, Albuquerque, New Mexico 87106

Received November 8, 2001. Revised Manuscript Received May 7, 2002

This study describes the transformation of cellulose acetate filters into porous carbon structures by a facile, direct process. Filters from cigarettes were used as the source for cellulose acetate filters. Carbon was obtained by high-temperature treatment under inert conditions without extensive preparative methods. The resulting carbon materials showed structural features on different length scales ranging from the micrometer to the nanometer scale. The materials were investigated using imaging techniques (scanning electron microscopy, SEM; transmission electron microcopy, TEM; and confocal Raman microscopy); the internal structure was investigated with Raman spectroscopy, wide-angleX-ray scattering (WAXS), small-angleX-ray scattering; and the porosity was explored using nitrogen sorption analysis.
\end{abstract}

\section{Introduction}

The various forms of elemental carbon have stimulated the research activities of scientists for many years. The three stable phases of carbon are graphite (thermodynamically stable at ambient conditions), diamond, and the fullerenes (metastable). Much of the work has focused on preparing different types (forms) of carbon materials from these modifications. The number of carbon forms, materials containing only elemental carbon, is therefore much higher than the number of carbon allotropes. In particular, it is desirable to prepare materials that are organized on a significantly larger scale than molecular or crystal unit-cell dimensions.

Materials containing hexagonal carbon layers (graphenes) show the greatest variety of forms. Graphite is characterized by extended $\mathrm{sp}^{2}$-carbon layers in a crystalline array. Various types of carbon such as coke, pyrolitic carbon, soot, and activated carbons are characterized by a graphene structure showing distortions and a lower degree of order than graphite. These structural features differ with respect to the ratio of $\mathrm{sp}^{2}$ to $\mathrm{sp}^{3}$-hybridized carbons, the crystallite size, the extension of graphene layers, the relative layer orientations, defects, etc. Numerous applications of these carbons as electrode materials, filters, or catalyst carriers are of technical interest.

In principle, every hydrocarbon can serve as a precursor for carbon by pyrolysis and other thermal treatments. In practice, only a few precursors give significant amounts of the desired carbons, and optimized heat treatment procedures must often be developed. Among

* Corresponding author. E-mail: spolarz@chem.utoronto.ca. Fax: (+1)-416-971 2011

† Max-Planck Institute of Colloids and Interfaces.

¥ University of Toronto.

$\S$ University of New Mexico. suitable precursors are sugars, acetylene, furfuryl alcohols, and phenol formaldehyde, whereas the earliest stages of carbonization are observed at temperatures of $\mathrm{T} \approx 400-500{ }^{\circ} \mathrm{C}$, depending on the chemical nature of the precursor.

Creating materials that are organized on different length scales (hierarchically or, according to Ozin, ${ }^{1,2}$ panoscopic) is a demanding task in modern materials chemistry. ${ }^{3,4}$ This challenge is especially true for carbon, because structure-forming techniques developed for ceramics, e.g., silica or metal oxides, 5,6 such as molding or sol-gel procedures ${ }^{7-9}$ are either unknown or difficult to apply for elemental carbon.

Among the different forms of carbon, porous carbon is very important because of the industrial relevance of charcoals originating from this porosity. Methods for obtaining porous carbons have been reviewed recently. ${ }^{10}$

Many procedures for the preparation of porous carbon involve sophisticated techniques and expensive and/or toxic chemicals. Additionally, panoscopic ${ }^{1}$ or hierarchically ordered materials are still quite rare. ${ }^{10}$

In this paper, a strategy for the preparation of porous carbons is presented that uses inexpensive precursors

(1) Ozin, G. A. Chem. Commun. 2000, 419.

(2) Chomski, E.; Ozin, G. A. Adv. Mater. 2000, 12, 1071. 325.

(4) Mann, S.; Ozin, G. A. Nature 1996, 382, 313.

(5) Raman, N. K.; Anderson, M. T.; Brinker, C. J . Chem. Mater. 1996, 8, 1682

(6) Ciesla, U.; Schüth, F. Microporous Mesoporous Mater. 1999, 27, 131

(7) Brinker, C. J .; Scherer, G. W. Sol-Gel Science: ThePhysics and Chemistry of Sol-Gel Processing, 1st ed.; Academic Press: New York, 1990.

(8) Barton, T. J .; Bull, L. M.; Klemperer, W. G.; Loy, D. A.; McEnaney, B.; Misono, M.; Monson, P. A.; Pez, G.; Scherer, G. W. Vartuli, J. C.; Yaghi, O. M. Chem. Mater. 1999, 11, 2633.

(9) Hench, L. L.; West, J. K. Chem. Rev. 1990, 90, 33.

(10) Kyotani, T. Carbon 2000, 38, 269. 


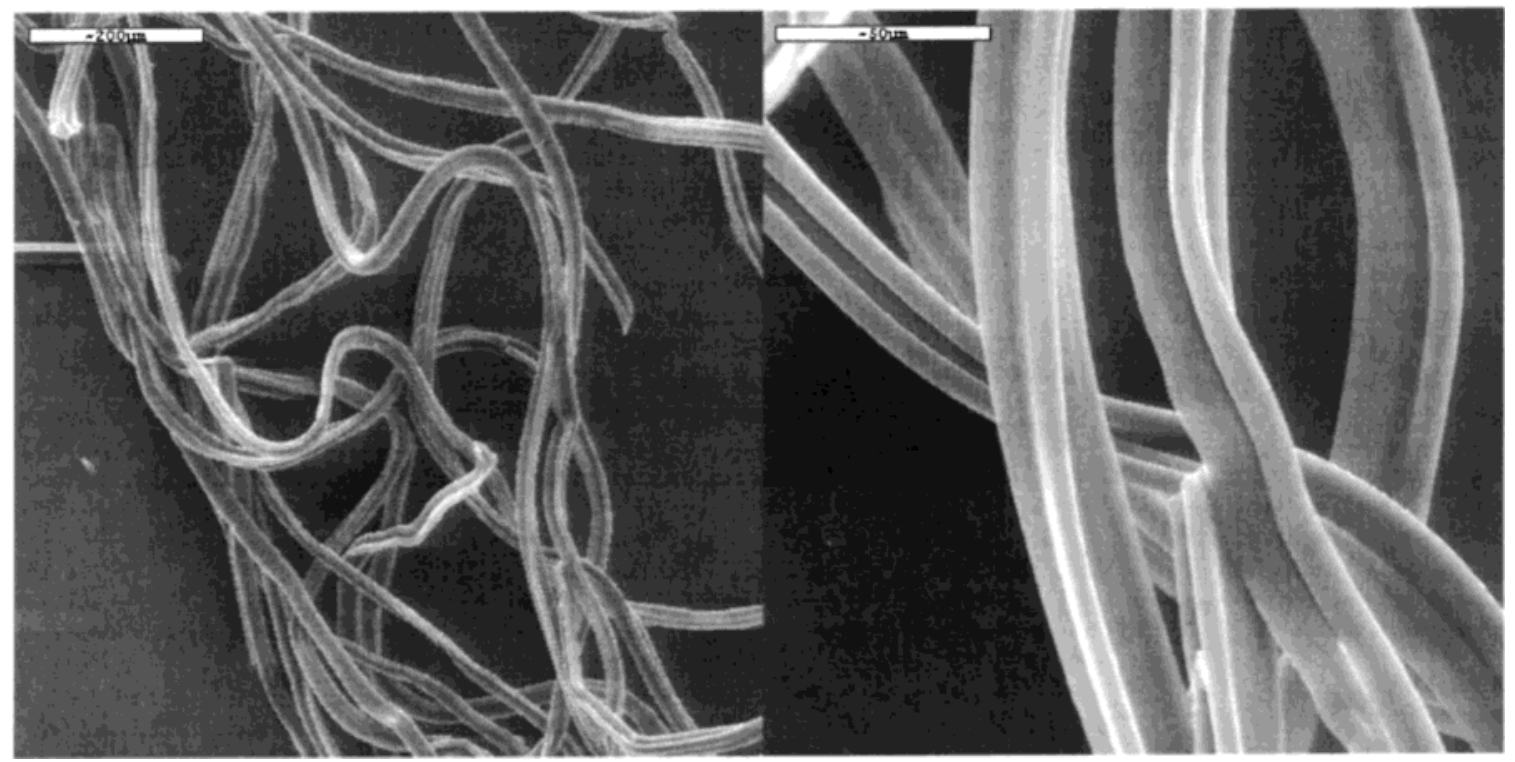

Figure 1. SEM images of cellulose acetate fibers originating from cigarette filters (prior to carbonization) at two different magnifications.

and is based on a simple one-step procedure. Cellul ose acetate precursors were chosen in the form of cigarette filters (smoked or unsmoked). The contribution of cigarette filters to solid waste is significant, and it is an important task of general interest to use waste as a new resource by suitable recycling procedures. Cellulose-containing organic waste was previously reported to provide a suitable precursor for carbonization. ${ }^{11-13}$ The number of cigarettes smoked per year $\left(\sim 1.5 \times 10^{4}\right.$ $\mathrm{t}$ in Germany) is immense, and the filters are thrown away in most cases.

Here, we show that the carbon structure derived from cellulose acetate is significantly different from the original morphology of the filter material and that the new carbon exhibits a hierarchical structure of different length scales ranging from the nanometer to the micrometer scale. The resulting carbon is investigated in composition and structure by various tedhniques, namely, Raman spectroscopy, confocal Raman mi croscopy, scanning electron microscopy (SEM), transmission el ectron microscopy (TEM), wide-angleX-ray scattering (WAXS), small-angleX-ray scattering (SAXS), nitrogen sorption analysis, thermogravimetric analysis (TGA), and elemental analysis.

\section{Experimental Section}

Preparation of Carbon. Smoked or unsmoked cigarettes were collected, and the coating was separated from the remaining filter material. Only pure filter material was used for carbonization studies. The filter material was transferred to a tube oven (Heraeus Thermicon $\mathrm{P}$ ) equipped with a quartz tube. The tube is flooded with nitrogen prior to slow heating $\left(1{ }^{\circ} \mathrm{C} / \mathrm{min}\right.$ ) to the final temperature of $800^{\circ} \mathrm{C}$. The sample was held at this temperature for $7 \mathrm{~h}$ under a continuous gas flow.

Analytical Techniques. Raman spectra and Raman images were obtained with a confocal Raman microscope from Witec. The Raman instrument was equipped with a 15-mW laser source $(\lambda=532 \mathrm{~nm})$. The carbon sample was placed on

(11) Kim, D. Y.; Nishiyama, Y.; Wada, M.; Kuga, S. Cellulose 2001 8,29

(12) Lim, S. K.; Cho, K. M.; Tasaka, S.; Inagaki, N. Macromol. Mater. Eng. 2001, 286, 187.

(13) Laszlo, K.; Bota, A.; Nagy, L. G. Carbon 2000, 38, 1965. a microscope slide and covered with a glass cover. Direct scattering was detected using a CCD detector system. For imaging, a significant Raman band was selected and deflected on an APD detector. The scattering was detected as a function of the spatial coordinates. The maximum imaging area was $200 \times 200 \mu \mathrm{m}^{2}$, and the maximum resolution was $200 \mathrm{~nm}$. Nitrogen sorption $\left(T=-196^{\circ} \mathrm{C}\right)$ data were obtained using a Micromeritics Tristar apparatus. The samples were dried at $150{ }^{\circ} \mathrm{C}$ in a vacuum before measurement. F or SEM (Zeiss DSM 940 instrument) analysis, the samples were placed on carboncoated stubs and sputter coated. TEM images were acquired with a Zeiss EM $912 \Omega$ instrument at an acceleration voltage of $120 \mathrm{kV}$. Samples were ground in a ball mill and taken up in acetone. One droplet of the suspension was applied to a 400mesh carbon-coated copper grid and left to dry in air. TGA measurements were performed with a Netzsch TG209 cell. WAXS patterns were collected with an Enraf Nonius FR590 diffractometer. Ball-milled samples were irradiated for approximately $5 \mathrm{~h}$. Small-angle X-ray scattering (SAXS) measurements were conducted with a Nonius rotating anode $(P=$ $4 \mathrm{~kW}, \mathrm{Cu} \mathrm{K} \alpha$ ) and an image-plate detector system. With the image plates placed at a distance of $40 \mathrm{~cm}$ from the sample, a scattering vector range of $\mathrm{s}=0.05-1.6 \mathrm{~nm}^{-1}$ was available (with $\mathrm{s}=2 / \lambda \sin \theta$, where $2 \theta$ is the scattering angle). The samples were irradiated for $18 \mathrm{~h}$ to reduce the noi se level and to obtain a sufficiently high scattering intensity. 2D diffraction patterns were transformed into 1D radial averages of the scattering intensity. Elemental analysis was performed using an Elementar Vario EL apparatus.

\section{Results and Discussion}

First, the morphol ogy of the cellulose acetate starting materials was investigated prior to carbonization. The filter morphology was investigated by SEM (see Figure 1 ). It is seen that a cigarette filter contains bundles of cellulose acetate fibers.

After heat treatment, it is observed that the cellulose acetate filter is transformed into a black carbon-like material. The pyrolyzed material is brittle and less dense than the original filter. The carbonization process was initially investigated by TGA. TGA indicates that the transformation to carbon via dehydration starts at a temperature of $\mathrm{T} \approx 280{ }^{\circ} \mathrm{C}$ and is completed at a temperature of $\mathrm{T}=360{ }^{\circ} \mathrm{C}$. The dehydration and removal of acetic acid causes a mass loss of $\sim 65 \%$, a 


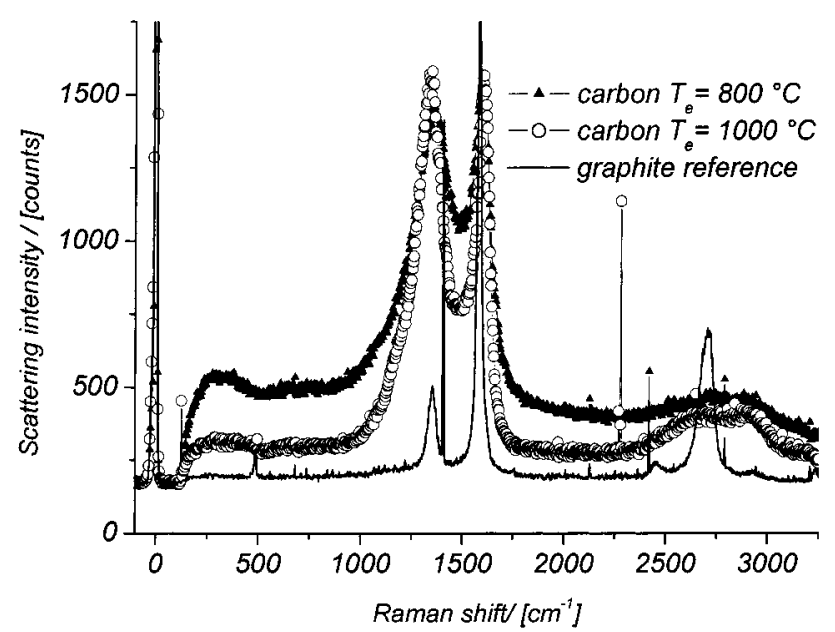

F igure 2. Raman spectrum of cellul ose acetate-based carbon materials produced at two different final temperatures $T_{e}$ and commercial graphite powder as a reference. The typical signals for graphite-related carbon are present for the prepared samples. The broadening of the Raman signals and a change in relative intensities is more evident for the ill-defined graphitic structure than for the commercial graphite.

value that is in good agreement with the expected relative amount of $\mathrm{H}_{2} \mathrm{O}$ and acetic acid to be removed from the sample. In the subsequent heat treatment up to a temperature of $\mathrm{T}=600^{\circ} \mathrm{C}$, a further mass loss of $20 \%$ is observed, possibly due to an incomplete degree of carbonization ( $\mathrm{C}_{\mathrm{x}} \mathrm{H}_{\mathrm{y}}$ species). Therefore, the carbon yield can be estimated to be $10-15 \%$, which is in good agreement with corresponding values reported in the literature. ${ }^{13}$ At higher temperatures, no significant mass change was detected. Elemental analysis of the pyrolyzed filter revealed the following composition of the material: $98 \% \mathrm{C}, 0.67 \% \mathrm{~N}_{2}$, and $0.1 \% \mathrm{H}$. Oxidation leaves $1.23 \%$ of inorganic salts behind, which were not further analyzed. The presence of large fractions of carbohydrates or other organic impurities can be excluded. Further indications for the complete transformation to elemental carbon are suggested by the Raman spectra (see Figure 2).

The occurrence of the two bands at $\bar{v}_{1}=1583 \mathrm{~cm}^{-1}$ and $\bar{v}_{2}=1344 \mathrm{~cm}^{-1}$ as found for the reference sample (synthetic graphite powder obtained from Aldrich), is typical of elemental carbon. These two signals are also observed in the case of the cellulose acetate-derived carbon materials (for two different carbonization temperatures, $\mathrm{T}_{\mathrm{e}}=800$ and $1000^{\circ} \mathrm{C}$ ) at the positions $\bar{\nu}_{1}=$ $1598 \mathrm{~cm}^{-1}$ and $\bar{v}_{2}=1351 \mathrm{~cm}^{-1}$. The small shift is within the range of error for the Raman setup used in this study and is not significant. Additionally, the graphite reference sample has a clear signal at $\bar{v}_{3}=2708 \mathrm{~cm}^{-1}$. This signal is heavily broadened (peak width of $\sim 1000$ $\mathrm{cm}^{-1}$ ) for the prepared carbon samples and clearly shifted to $\bar{v}_{3}=2828 \mathrm{~cm}^{-1}\left(\Delta \bar{v}=120 \mathrm{~cm}^{-1}\right)$. The peaks at $\bar{v}_{1}$ and $\bar{v}_{2}$ are also broadened, but to a lesser degree. This broadening is possibly caused by an uncertainty in energy for the corresponding vibrations. These observations indicate that the prepared carbon material shows a structure of 2D hexagonal carbon layers (graphenes) that is significantly less defined than is observed for graphite. Further information can be obtained from the vibrations corresponding to $\bar{\nu}_{1}$ and $\bar{v}_{2} . \bar{v}_{1}$ bel ongs to the $\mathrm{A}_{1 \mathrm{~g}}$ totally symmetric disorder mode

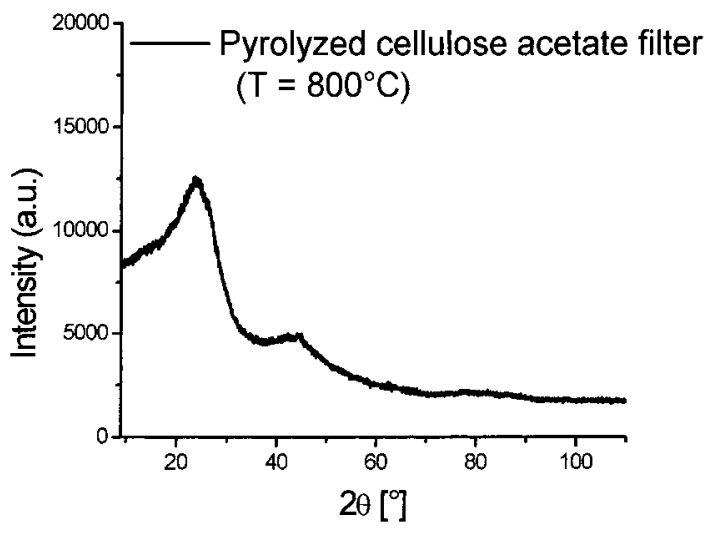

Figure 3. WAXS diffractograms of the carbon material resulting from thermal treatment of cellulose acetate fibers. The positions and shapes of the diffraction signals are indicative of turbostratic carbon.

(d mode) occurring in sputtered or diamond-like carbons. ${ }^{14} \bar{\nu}_{2}$ corresponds to the active $\mathrm{E}_{2 \mathrm{~g}}$ line of singlecrystall ine graphite (g mode). The parameter $\mathrm{L}_{a}$, which describes the average extension of the graphenes, can be estimated from these data by the intensities of the Raman $\mathrm{d}$ and $\mathrm{g}$ bands as reported by Tuinstra and Koenig ${ }^{15,16}$

$$
\mathrm{L}_{\mathrm{a}}=(4.35 \mathrm{~nm}) \times \frac{\mathrm{I}_{\mathrm{g}}}{\mathrm{I}_{\mathrm{d}}}
$$

The parameter $\mathrm{L}_{a}$ in the carbon material with $\mathrm{T}_{\mathrm{e}}=800$ ${ }^{\circ} \mathrm{C}$ thus can be estimated as $\mathrm{L}_{\mathrm{a}} \approx 4.6 \mathrm{~nm}$. An increase in carbonization temperature leads to several changes. It can be seen from Figure 2 that the d peaks have nearly the same intensities for the samples prepared at $\mathrm{T}_{\mathrm{e}}=1000$ and $800^{\circ} \mathrm{C}$, but the g peak clearly increases in relation to the $d$ peak. Additionally, the peaks at $T_{e}$ $=1000{ }^{\circ} \mathrm{C}$ are sharpened. No peaks that do not correspond to elemental carbon are present; in particular, no peaks for cellulose acetate are observed. This supports the conclusion that the thermal treatment process produced a pure nongraphitic carbon without a substantial degree of chemical impurities. According to previous studies, amorphous carbon materials without a graphene structure show a broad Raman band at $\bar{v}=$ $1530 \mathrm{~cm}^{-1} \cdot{ }^{17}$ This band is not present in our case. Hence, the Raman data indicate that the carbon material belongs to the group of nongraphitic carbons with 2D graphenes constituting the basic structural unit. Wideangle X-ray scattering (WAXS) was carried out to further describe the structure of this carbon material on the nanometer scale, in particular, to determine size and disorder parameters. The diffraction pattern (see Figure 3 ) shows three major signals at the positions $24^{\circ}$ (002), $44^{\circ}(10)$, and $\sim 83^{\circ}$ [superposition of various (hk) and (00l) interferences]. These signals are typical of nongraphitic carbons with a significant degree of rotational and translational disorder in the stacking of the graphenes (turbostratic carbon).

In addition to the determination of the average extension $L_{a}$ of the graphene layers from the (hk)

(14) Matsunuma, S. Thin Solid Films 1997, 306, 17

(15) Tuinstra, F.; Koenig, J . L. J . Chem. Phys. 1970, 53, 1126.

(16) Tuinstra, F.; Koenig, J . L. Bull. Am. Phys. Soc. 1970, 15, 296.

(17) Zerda, T. W.; Xu, W.; Zerda, A.; Zhao, Y.; Von Dreele, R. B. Carbon 2000, 38, 355. 
a)

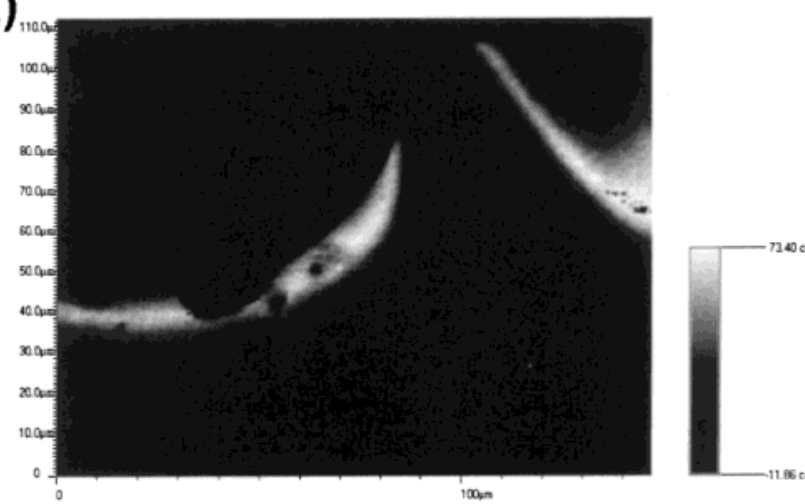

b)

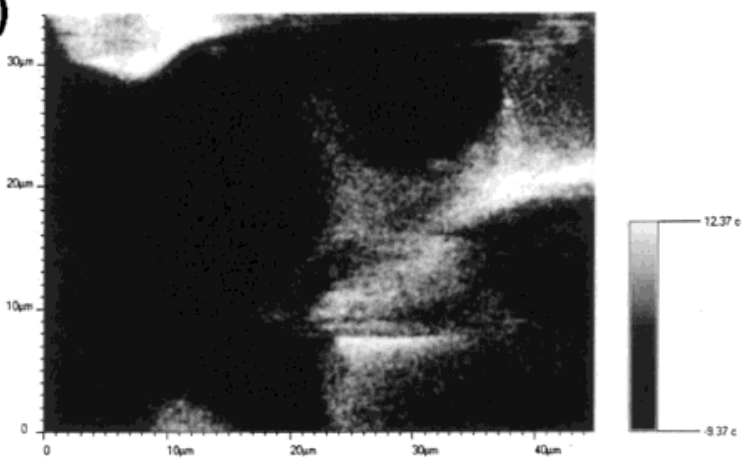

Figure 4. Confocal Raman images of two sections of the prepared porous carbon material. The imaging was done using the Raman band at $1583 \mathrm{~cm}^{-1}$. Therefore, the figure shows the spatially resolved distribution of carbon in the sample. Regi ons with (a) larger and (b) smaller macropores are shown.

interferences, it is possible to estimate the average stack size $L_{c}$ from the $(00 \mathrm{l})$ interferences by previously introduced evaluation procedures. ${ }^{18,19}$ Both the small value for $L_{a}$ of $4.6 \mathrm{~nm}$ and the large integral width of the (10) interference indicate a significant degree of disorder in the 2D lattice. From the evaluation of the interlayer interferences, the average stack size $L_{c}$ was determined to be about $3 \mathrm{~nm}$. Together with an average interlayer spacing of $a_{3}=0.357 \mathrm{~nm}$ with a variance $\sigma_{3}=0.45 \mathrm{~nm}$, the carbon also shows a significant degree of disorder in the layer stacking. In particular, from the detailed analysis of the WAXS data, it can be concluded that the precursor is nearly completely transformed into turbostratic carbon, that is only a small fraction ( $<5 \mathrm{wt} \%)$ of the precursor is transferred into "unorganized carbon" or other non-sp ${ }^{2}$ species.

In the following, we consider the structure of the carbon at larger length scales. An initial presumption of the morphology of the carbon can be obtained by Raman imaging with confocal microscopy. To do so, the most intense signal from the Raman spectrum is chosen $\left(\bar{v}_{1}\right)$ and detected as a function of 2D spatial coordinates. One obtains a two-dimensional image of the sample with the signal intensity (color-coded) as the third dimension (see Figure 4).

It is seen that the morphology of the carbon material differs substantially from that of the cellulose acetate fiber starting material (compare Figures 1, 4, and 5). The prepared samples show curved and extended areas

(18) Ruland, W.; Smarsly, B. J . Appl. Crystallogr. 2002, in press. (19) Gregg, S. J .; Sing, K. S. W. Adsorption, Surface Area and Porosity, 4th ed.; Academic Press: New York, 1982; Vol. 2.

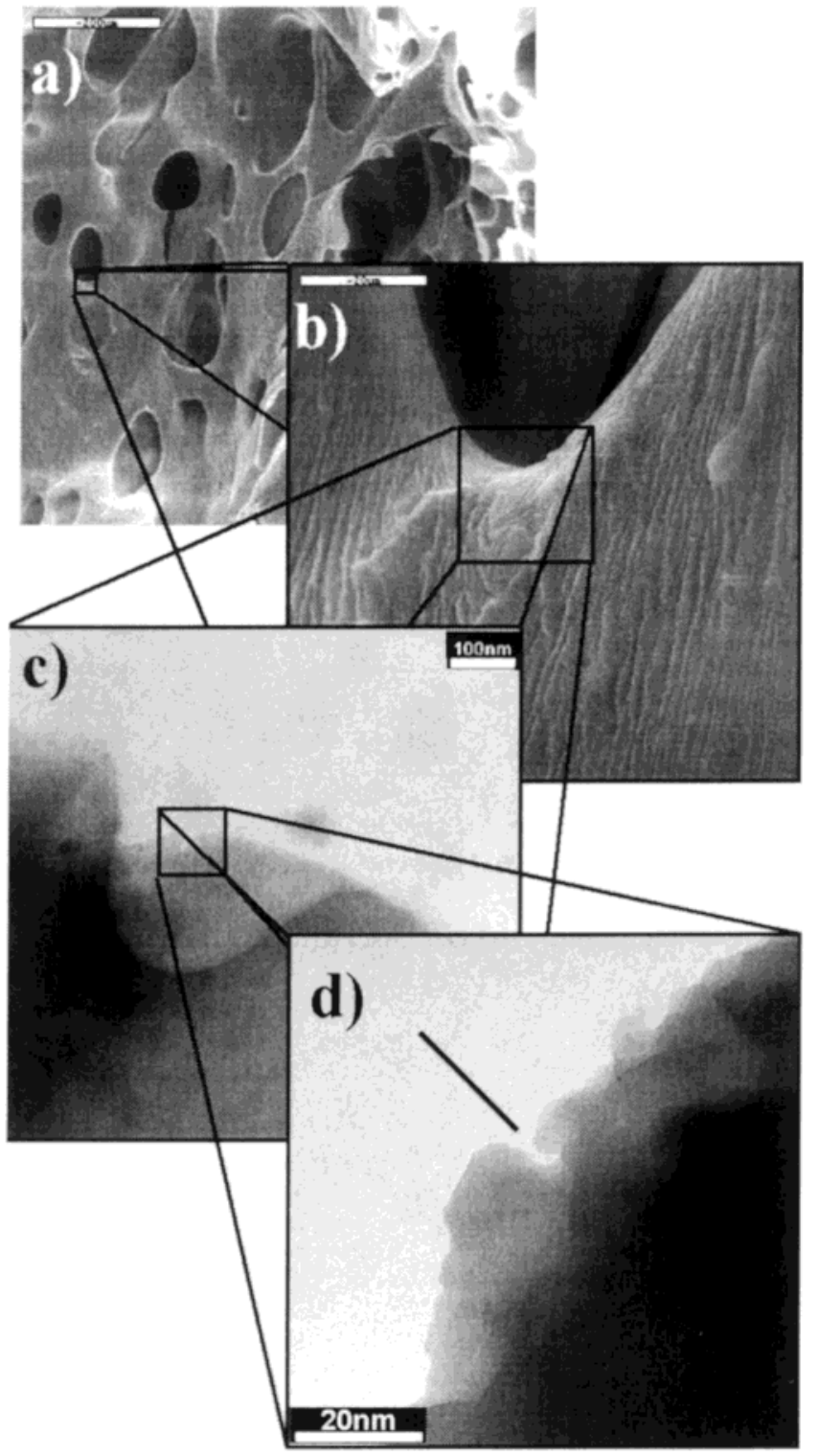

Figure 5. SEM and TEM images of the resulting carbonmaterials: I nvestigation of different length scales. I mage a shows how large macropore are interconnected in a carbon network. At higher magnifications, this network exhibits a lamellar substructure, as shown in b. In image c, a different area of the sample was measured with TEM. At higher magnifications, mesopores appear, as indicated by an arrow in image $d$. The mode of representation was chosen to demonstrate the hierachical structure of the material.

that suggest a porous material. According to the Raman images, the pore sizes are in the range of several micrometers. The macropore size distribution is very broad as a result of large cavities ( $>50 \mu \mathrm{m}$ as indicated by Figure 4a) and smaller porous domains with sizes of $\sim 10-30 \mu \mathrm{m}$ (Figure 4b). Although Raman images yield some morphology clues, a detailed description of the morphology is difficult to obtain in this case, because the maximum scanning area $\left(200 \times 200 \mu \mathrm{m}^{2}\right)$ is of the same order as the pore size.

Scanning electron microscopy (SEM) was applied to elucidate the formation of the carbon material from cellul ose acetate from a morphological point of view (see Figure $5 a, b)$. The results obtained from Raman imaging are supported by the SEM measurements. A macroporous carbon (Figure $5 a$ ) with a polydisperse pore size distri- 

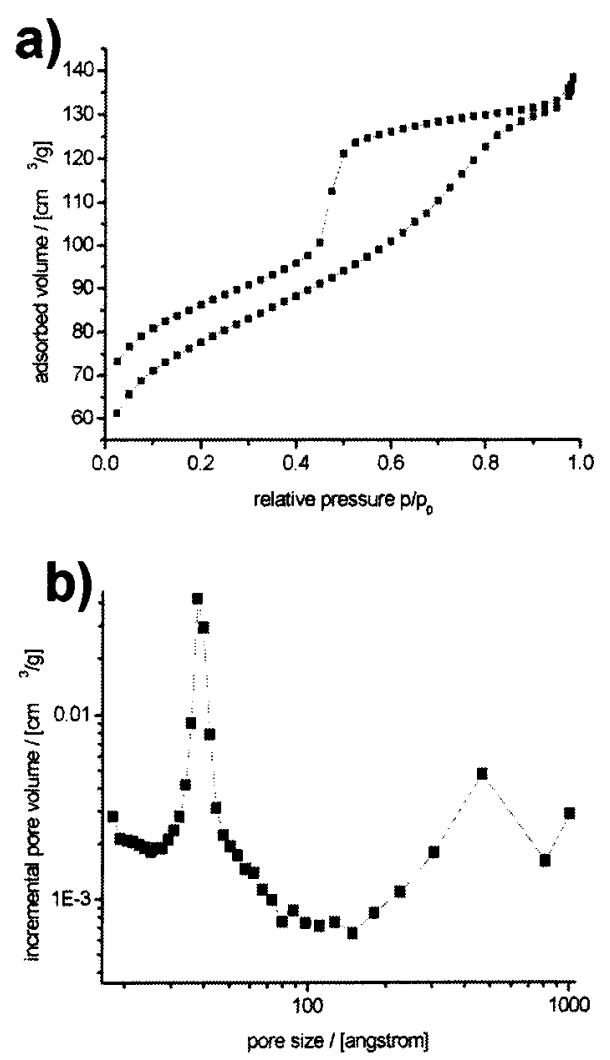

Figure 6. (a) $\mathrm{N}_{2}$ isotherms of the prepared carbon materials and (b) the corresponding pore size distribution.

bution, ranging from about $20-200 \mu \mathrm{m}$, is seen. The pores are randomly distributed in the carbon network, but they appear to be interconnected. At higher magnifications (Figure $5 b$ ), a lamellar substructure can be identified in the carbon network. The thickness of the layers in the lamellae can be estimated as $d \approx 150-$ $200 \mathrm{~nm}$. To elucidate the structure on the nanometer scale, the carbon material was also investigated by transmission el ectron microscopy (TEM).

TEM samples were acquired from ground samples and a position from a small macropore was chosen for the image. It is seen in Figure $5 c$ that the rim of the macropore seems to host smaller pores in the mesopore region. These pores are quite small (radius $=2-4 \mathrm{~nm}$, length $\approx 5-10 \mathrm{~nm}$; Figure $5 \mathrm{~d}$ ) and might result from activation processes that occur during carbonization.

The mesoporosity was further investigated by $\mathrm{N}_{2}$ sorption analysis to determine the mesopore volume and the mesopore size distribution (see Figure 6).

The typical features of sorption of mesoporous materials ${ }^{19-21}$ can be identified in the $\mathrm{N}_{2}$ isotherm shown in Figure 6a. At $\mathrm{p} / \mathrm{p}_{0}=0.6$, a capillary condensation step is clearly visible although it is not strongly expressed, indicating a certain degree of polydispersity in the mesopore size. From the sorption data, a specific surface area of $262 \mathrm{~m}^{2} / \mathrm{g}$ (BET) is obtained, along with a porosity of $0.21 \mathrm{~cm}^{3} \mathrm{~m} / \mathrm{g}$. The sorption in large macropores is visible in the isotherm at high pressures

(20) Rouqerol, J .; Avnir, D.; Fairbridge, C. W.; Everett, D. H.; Haynes, J . H.; Pernicone, N.; Ramsay, J . D.; Sing, K. S. W.; Unger, K. K. Pure Appl. Chem. 1994, 66, 1739.

(21) Sing, K. S. W.; Everett, D. H.; Haul, R. A. W.; Moscou, L.; Pierotti, R. A.; Rouquérol, J .; Siemieniewska, T. Pure Appl. Chem 1985, 57, 603. $\left(p / p_{0} \rightarrow 1\right)$, where the adsorbed volume again increases. The isotherm exhibits a clear hysteresis typical of mesoporous systems. However, the observed hysteresis is quite broad, which is a sign of a pore system with a distinct polydispersity in size, thus supporting the TEM results discussed above. Interestingly, the adsorption and desorption branches of the isotherm do not coincide at small $\mathrm{p} / \mathrm{p}_{0}=0$. This phenomenon is known for certain carbon materials and might be due to changes in the bulk density upon nitrogen sorption or the irreversible adsorption of nitrogen. ${ }^{13}$ Moreover, this effect might also be connected with retarded adsorption, where the adsorbing and desorbing molecules have to overcome a certain energy barrier, which might be a consequence of the presence of narrow pores, penetration into which is difficult because of steric considerations. ${ }^{22,23} \mathrm{From}$ the adsorption branch of the isotherm, a pore size distribution (using a DFT approach) ${ }^{24}$ was evaluated that yielded a distinct maximum at a pore diameter of 4.5 $\mathrm{nm}$, assuming a cylindrical pore morphology. Moreover, the pore size distribution indicates the presence of larger pores in the range of $40-90 \mathrm{~nm}$. Hence, the pyrolyzed cellulose acetate material shows a pronounced mesoporosity with a preferential mesopore size. It is interesting to note that, in previous studies on the carbonization of cellulose materials, the mesopores were of significantly different sizes. Laszlo et al. described cellulosebased carbons with mesopore sizes of ca. $3 \mathrm{~nm}$, and al so in this case, the authors observed quite a narrow size distribution. ${ }^{13}$ Hence, our studies confirm the observation that cellulose-derived materials produce a more developed mesoporosity than other polymeric precursors such as polyacrylonitrile. To further investigate the porosity, small-angle X-ray scattering (SAXS) was employed. Average pore sizes can be determined from SAXS data by previously reported methods ${ }^{25,26}$ using the relationship

$$
\frac{1}{I_{p}}=\frac{1}{I_{\text {pore }}}+\frac{1}{I_{\text {solid }}}=\frac{1}{\phi I_{\text {solid }}}=\frac{1}{(1-\phi) I_{\text {pore }}}
$$

where $\phi$ is the vol ume fraction of pores; $I_{p}$ is the "Porod length"; and I pore and $I_{\text {solid }}$ are the average sizes of the pores and the solid material, respectively. $I_{p}$ is available accurately from SAXS data (not shown here) by recently published methods, ${ }^{25-27}$ and $\phi$ was determined from nitrogen sorption measurements (assuming that all of the mesopores were accessible). An example of this method is given in detail in ref 27 . We obtained a value of $I_{p} \approx 2 \mathrm{~nm}$, which corresponds to an average pore size of $4 \mathrm{~nm}$. This value is slightly less than the average mesopore size obtained from nitrogen sorption, which might be because of the presence of some microporosity or the assumption of cylindrical pores in the sorption analysis.

(22) Rodriguez-Reinoso, F.; Linares-Solano, A. In Chemistry and Physics of Carbon; Thrower, P. A., Ed.; Marcel Dekker: New York, 1989; $\mathrm{p} 1$.

(23) Marsh, H.; Wynne-J ones, W. F. K. Carbon 1964, 1, 281.

(24) Neimark, A. V.; Ravikovitch, P. I. Stud. Surf. Sci. Catal. 2000 128,51 .

(25) Mering, P.J.; Tchoubar, D. J . Appl. Crystallogr. 1968, 1, 153.

(26) Perret, R.; Ruland, W. J. Appl. Crystallogr. 1968, 1, 308. 2618. 


\section{Conclusions}

In the present work, the use of cellulose acetate waste in the form of cigarette filters as a precursor for porous carbon material is described. The carbon obtained via pyrolysis is nearly completely transformed into nongraphitic carbon and shows a complex pore structure. The material exhibits a well-defined mesoporosity with an average pore diameter of 4-5 $\mathrm{nm}$ and a relatively narrow size distribution, thus confirming previous studies on cellulose. Moreover, a hierarchical complex pore morphology is also observed on higher length scales, namely, in the range of $100 \mathrm{~nm}$ up to the micrometer domain. Because a corresponding change in the microstructure does not seem to occur with pure cellul ose as a carbon precursor, the development of a hierarchical pore structure during the carbonization process could be a consequence of the modification by acetate. This study indicates that complex biomolecules, such as cellulose, that exhibit hierarchical supramolecular structures between the nanometer and micrometer range are able to provide porous carbons that also exhibit welldefined porosities on different orders of magnitude. It should be emphasized that the morphology of the resulting carbon materials is quite different from that of the cellulose acetate fibers used as the precursor (compare Figures 1 and 5). Cellulose is a thermoplastic material; thus, the cellulose fibers can be seen as stretched cellulose. U pon heat treatment above the glass transition, the fibers will contract, and at a certain stage, melting will occur. Simultaneously, carbonization occurs and fixes a certain stage of the thermoplastic rearrangement of the cellulose that is, for instance, seen in the lamellar structure. A similar alternative could be a transformation via lamellar mesophases, as reported for the transformation of polyacrylonitrile to carbon by Thünemann and Ruland in 2000.28

These materials could find applications in the field of gas purification or more generally as filters.

Acknowledgment. Dr. J. Hartmann is acknowledged for the SEM measurements of the cellulose acetate fibers. We thank the Max-Planck society for funding.

CM011271R

(28) Thünemann, A. F.; Ruland, W. Macromolecules 2000, 33, 2626. 